\title{
DESIGN OF DISSOLUTION STUDY PROTOCOL FOR PULMONARY DOSAGE FORMS: CRITERIA FOR SELECTION OF BIO-RELEVANT DISSOLUTION MEDIUM
}

\author{
SAUMYAJYOTI DAS*, PRASENJIT SARKAR, SUTAPA BISWAS MAJEE
}

Department of Pharmaceutical Technology, Division of Pharmaceutics, NSHM Knowledge Campus, Kolkata-Group of Institutions, Kolkata, West Bengal, India. Email: dassaumya751@gmail.com

Received: 14 December 2021, Revised and Accepted: 07 January 2022

\begin{abstract}
Pulmonary dosage forms constitute an important route of drug delivery for systemic absorption of drugs in management of respiratory diseases as well as diseases such as diabetes, migraine, osteoporosis, and cancer. Performance of different pulmonary dosage forms is greatly influenced by aerodynamic particle size distribution of inhalable particles, spray pattern, fraction of dose actually deposited on pulmonary epithelium, dissolution of active pharmaceutical ingredient and ultimately absorption across pulmonary barriers. In vitro dissolution study should be designed to predict in vivo performance precisely, providing key information on bioavailability and establishing in vitro-in vivo correlation. To obtain meaningful data from dissolution study, focus should be on composition of dissolution medium, dissolution conditions and dissolution test apparatus. For pulmonary dosage forms, selection of physiologically relevant dissolution medium, mimicking lung fluid (LF) is a challenging task. Attempts are being made to develop bio-relevant dissolution medium to overcome the limitations associated with use of conventional media lacking lung surfactant proteins, or several salts normally present in pleural fluid. Use of simulated LFs can give a better understanding of the release mechanisms and possible in vivo behavior of pulmonary dosage forms thereby enhancing the predictive capability of the dissolution testing. In the review, efforts have been taken to provide comprehensive information on composition, physicochemical characteristics and functions of physiological LF, challenges associated with the design and development of dissolution study protocol for pulmonary dosage forms, criteria for selection of an appropriate bio-relevant dissolution medium, comparative study on various reported bio-relevant dissolution media and dissolution apparatuses employed for in vitro characterization of performance of pulmonary dosage forms.
\end{abstract}

Keywords: Airway surface liquid, Andersen cascade impactor, Bio-relevant dissolution medium, Dipalmitoyl phosphatidylcholine, DissolvIt, Lung surfactant protein, Next generator impactor, Pulmonary dosage forms, Simulated lung fluid, Transwell.

(C) 2022 The Authors. Published by Innovare Academic Sciences Pvt Ltd. This is an open access article under the CC BY license (http://creativecommons.org/ licenses/by/4.0/) DOI: http://dx.doi.org/10.22159/ajpcr.2022v15i2.43887. Journal homepage: https://innovareacademics.in/journals/index.php/ajpcr

\section{INTRODUCTION}

Drug delivery into the lungs through pulmonary route has become one of the most important aspects of local as well as systemic drug delivery [1]. The origin of inhalation therapy can be traced back to India 4000 years ago where people smoked the leaves of Atropa belladonna to suppress cough. Drug delivery to the respiratory tract is principally performed for the treatment of local disorders, for example, asthma and cystic fibrosis. The pulmonary route can be employed to achieve drug absorption into systemic circulation without direct passage to liver thereby avoiding the hepatic first-pass effect [2-4]. Moreover, drug delivery by this route can be considered ideal for systemic absorption of drugs owing to excellent blood perfusion of lungs and large surface area provided by the alveoli. The pulmonary route presents several advantages in the treatment of respiratory diseases (e.g., asthma, chronic obstructive bronchopneumopathy) and as well as in some other indications such as diabetes, migraine, osteoporosis, and cancer over the administration of the same drugs by other routes. Despite facing challenges such as mucociliary clearance, short residence time, need for particle size optimization, etc., research is going on to develop technology to deliver drugs through pulmonary route $[5,6]$.

There are three main types of delivery systems for respiratory dosage forms which include metered-dose inhalers (MDIs), dry-powder inhalers (DPIs), and nebulizers. MDIs are pressurized hand-held devices that use propellants for delivering micronized drug in either dissolved or suspended state together with other excipients including surfactants and presented in a pressurized canister fitted with metering valve. In propellant-free DPIs, drug is inhaled as mist of fine particles. The drug is either preloaded in an inhalation device or filled into hard gelatin capsules or foil blister discs, which are loaded into a device before use.
DPIs are being increasingly popular with the growing concerns over the use of chlorofluorocarbons as propellant in MDIs. Nebulizers are devices for converting aqueous solutions preferably or micronized suspensions of drugs into an aerosol for inhalation. Nebulizers are frequently used for drugs that cannot be conveniently formulated into MDIs or DPIs [7].

For in vitro performance testing of pulmonary dosage forms, dose content uniformity and particle size distribution are the most important parameters that need to be monitored. Delivery of API from a specified delivery device and its deposition using a pharmaceutical impactor on artificial surface or membrane also needs to be investigated to estimate the actual dose delivered to lungs. Thus, an ideal dissolution test procedure for inhalation formulations should involve particle classification followed by evaluation of dissolution profile from sorted drug particles that may deposit at various sites in respiratory tract. Some of the commonly used media for dissolution testing of inhaled products include distilled deionized water, phosphate buffered saline (PBS) (pH 7.4), mixture of citrate buffer and ascorbic acid ( $\mathrm{pH}$ 5.2), mixture of water and $0.5 \%$ sodium dodecyl sulfate (SDS), etc. However, the media mentioned so far have little physiological relevance with the actual lung fluid (LF) thus failing to achieve in vivo dissolution profile predictive of in vivo inhaled drug products. To overcome this problem, bio-relevant dissolution media should be developed for better predictability of in vivo absorption profile of pulmonary dosage forms [8-18].

The objective of the review is to provide comprehensive information on the need for the development of suitable bio-relevant dissolution media to predict and characterize in vivo dissolution behavior of pulmonary dosage forms (PDFs). No article could be found in literature describing 
various simulated LFs used till date for characterization of in vitro drug release profiles from inhaled products and benefits of the use of such bio-relevant media over conventional ones. Ability of the media to mimic the physiological LF closely can enhance the in vivo predictability of drug dissolution studies. For better understanding of need for bio-relevant dissolution media and their selection, it is essential to have sufficient knowledge of lung physiology, LFs, surfactant and also barriers to pulmonary absorption which have been aptly described here.

\section{ANATOMY AND PHYSIOLOGY OF LUNGS}

Anatomically, the respiratory system consists of two parts, the upper and the lower respiratory tract. The upper respiratory tract located outside the chest cavity is composed of nose, pharynx, and the larynx. The lower respiratory tract which is located inside the chest cavity is composed of trachea, the bronchi, the bronchioles, and the alveoli, which are the functional units of lungs. The lungs lie on either sides of the mediastinum, within the thoracic cavity. Each lung is surrounded by a pleural cavity, which is formed by the visceral and parietal pleura. The lungs are made up of 300 million alveoli, highly vascularized by 280 billion pulmonary capillaries which provide a vast exposed surface area of 50-100 $\mathrm{m}^{2}$ for effective gaseous exchange. Large surface area, good vascularization, thinness of alveolar epithelium $(0.1-0.2 \mu \mathrm{m})$ and high permeability of lungs render them ideal for drug delivery. With respect to pulmonary drug delivery the respiratory tract can be divided into three regions namely nasopharyngeal (NP) region, tracheobronchial (TB) region, and alveolar region (A). The NP region includes the respiratory airways from nose down to larynx, TB region starts at larynx and extends via trachea, bronchi, and bronchioles and ends at terminal bronchioles and alveolar region comprises of respiratory bronchioles, alveolar ducts and alveoli. The alveoli consist of single cell layer of squamous epithelium containing highly vascularized capillaries [19-26]. There are mainly two types of cells present in alveolar epithelium termed as type 1 pneumocyte responsible for gaseous exchange and type 2 pneumocyte cells which produce, secrete and recycle pulmonary surfactant proteins [SP].

\section{Lung fluid (LF): Physicochemical properties}

The lung or pleural fluid is a clear ultrafiltrate of plasma that originates from the parietal pleura. The ion content of human pleural fluid indicates that it is significantly hypo-osmotic $(222 \mathrm{mOsm})$ in relation to plasma. The $\mathrm{pH}$ of healthy LF has been reported to be 7.3-7.4. About 10-30 $\mathrm{ml}$ of LF termed as airway surface liquid (ASL) covers the wide surface area of lungs that lies between the airway epithelium and the gas in the lumen which can protect the alveolar surface and bronchioles from external damage thereby providing the first line of defense. ASL ensures muco-ciliary transport of inhaled particles. Basically the ASL is composed of upper viscous mucus layer followed by lower periciliary liquid layer. The thickness of these two layers together is in range of 5-20 $\mu \mathrm{m}$ in healthy individuals. The surface tension of ASL in healthy individual is $<10 \mathrm{mN} / \mathrm{m}[27,28]$. The various ions present in the ASL include Sodium $\left(\mathrm{Na}^{+}\right)$, Potassium $\left(\mathrm{K}^{+}\right)$, Magnesium $\left(\mathrm{Mg}^{2+}\right)$, Calcium $\left(\mathrm{Ca}^{2+}\right)$, Chloride $\left(\mathrm{Cl}^{-}\right)$, Sulfate $\left(\mathrm{SO}_{4}^{2-}\right)$, Bicarbonate $\left(\mathrm{HCO}_{3}^{-}\right)$, Acetate $\left(\mathrm{CH}_{3} \mathrm{COO}^{-}\right)$, and Phosphate $\left(\mathrm{PO}_{4}{ }^{2-}\right)$ ions. It is necessary for the inhaled drug particles to get dissolved in respiratory tract lining fluid (RTLF) covering the respiratory tract epithelial cells from nasal mucosa to alveoli. RTLF is composed of $95 \%$ water, $1 \%$ proteins, $2 \%$ mucins (glycoproteins [GP]), and 1\% inorganic salt.

\section{Lung surfactant (LS): Physicochemical properties and functions} The ions and surfactants of the ASL mainly control the surface tension. The surfactants in ASL consist of proteins (surfactant-specific proteins [SP] or serum proteins, such as albumin, fibrin, and transferrin) which are associated to the phospholipids. Around $92 \%$ of LS is constituted by lipid and $8 \%$ is constituted by the SP. The major phospholipid in LS is dipalmitoyl phosphatidylcholine (DPPC), although it may contain unsaturated PC, phosphatidyl glycerol (PG), and phosphatidyl inositol. Remaining 8\% LS includes four specific SP which are categorized into two families. The collectin protein family comprises of SP-A and SP-D,
SP-A being the most abundant hydrophilic GP. The smaller hydrophobic SP-B and SP-C are embedded in the LS associated with phospholipid layers. LS improves the mechanical stabilization of the lung alveoli thereby preventing the alveolar collapse. Moreover, LS also plays an important role in stabilizing the fluid balance in lungs thereby protecting the lungs against edema. LS can also enhance the solubility, promote the dissolution and absorption of poorly water soluble inhaled drugs due to its inherent capabilities of reducing interfacial tension $[29,30]$.

\section{BARRIERS TO ABSORPTION AND TRANSPORT MECHANISMS ACROSS PULMONARY EPITHELIUM}

There are mainly three types of barriers to drug permeation into alveoli such as alveolar-capillary barrier (air-blood barrier), interstitium, and vascular endothelium. Capillaries can be seen lying in a slightly asymmetrical fashion within alveolar walls, so that the alveolar-capillary membrane is on one side relatively thick and on the other side thin. The latter measures less than $0.5 \mu \mathrm{m}$ in cross section, and comprises an epithelial layer of type I alveolar cells, capillary endothelium, and between these two cell types lies a single basement membrane, which is a continuation of the alveolar basement membrane. The lung interstitium is the extracellular space between cells in tissue. In order for a molecule to be absorbed from the airspaces through the interstitium it must pass through the interstitium. Within the interstitium are fibroblasts, tough connective fibers and interstitial fluid which slowly diffuse and percolates through the tissue. The vascular endothelium is the final barrier to a molecule being absorbed from the airspace into the blood. Permeation of ions or inhaled drug molecules across the pulmonary epithelium follows specific mechanism to overcome the aforesaid barriers. The ion transport across airway epithelium occurs via transcellular route. The cells in airway epithelium are required to be polarized for the effective transport of ions. There are mainly four steps involved in the process of ion transport across the airway epithelium. Step 1 involves the active extrusion of sodium ions via Na-KATPase from the cell to the interstitium. This is followed by step 2 which involves the movement of anions to counteract the positive charge. Step 3 involves the accumulation of sodium ions and anions in the interstitial space to produce an osmotic gradient from lumen to interstitium that promotes water influx. In the final step, accumulation of salt and water occurs in the interstitium to promote the bulk flow of solute and water into the pulmonary capillaries. Some of the ion channels present on the apical and basolateral membranes of the air way epithelium include $\mathrm{Na}^{+}, \mathrm{Cl}^{-}$, and $\mathrm{K}^{+}$channels [31-40].

\section{IN VITRO QUALITY CONTROL (QC) TEST PARAMETERS FOR PULMONARY DOSAGE FORMS}

Various QC tests are performed on pulmonary aerosols and the propellants to guarantee that the ultimate product is at its optimum performance with the highest safety and that it fulfils product specifications and deliverables. Tests on propellants include determination of flammability and combustibility. Other tests on propellants include estimation of vapor pressure, density and moisture content. Aerosol valve discharge rate is determined by taking an aerosol product of known weight and discharging the contents for a given period of time using a standard apparatus. Spray pattern testing is based on the impingement of spray on a piece of paper that has been treated with a dye-talc mixture. Cascade impactor and light scatter decay methods are used for determination of particle size distribution (PSD) of aerosol. Other QC tests for aerosol include leak testing, foam stability testing, net content determination, etc. Delivered dose uniformity test is one of the important QC test applicable for MDI/DPI [41]. However, various other QC tests which are performed in case of MDI include physical characterization of drug substance and excipients, tests for minimum fill justification, actuator or mouth piece deposition, effects of environmental moisture on formulation, robustness, etc.

Two aspects which are primarily important in determining the in vivo performance of an inhaled formulation include total amount of dose deposited in the lung and its size distribution. The deposition is a 
consequence of the breathing pattern, the device used and the particle engineering. The dissolution profile of inhaled particles depends on the method used for collecting the amount of inhaled particles and their mass. Drug release is affected by the size of the particles collected after impaction (as the dissolution rate is inversely proportional to the radius of the particles, especially for particles within the range of 1.5-10 $\mu \mathrm{m}$ ), drug solubility, diffusion layer thickness, and particle shape $[41,42]$. Detailed information on different apparatuses used for dose collection and subsequent drug release testing has been provided in a later section.

\section{CHALLENGES TO DESIGN AND DEVELOPMENT OF DISSOLUTION STUDY FOR PULMONARY DOSAGE FORMS: SELECTION OF DISSOLUTION MEDIUM}

Dissolution testing is considered to be a valuable tool which can provide key information about bioavailability or bio equivalency as well as batch to batch consistency of any pharmaceutical dosage form. The selection of appropriate dissolution medium is a critical factor which depends on the purpose of study and drug molecule being investigated. It is advisable to select medium which can mimic more closely the biological fluid for prediction and modeling purposes. Limitations associated with the preparation of conventional media in study of dissolution profile of pulmonary dosage forms include increased risk of salt precipitation and use of citrate instead of protein, use of acetate replacing organic acids to avoid foaming which lead to deviation from original components in LF [43]. Although some of the dissolution media being used have some physiological relevance, these media are not capable to simulate the LF properly. In some cases, these media exhibit insufficient buffering effect leading to inappropriate prediction of dissolution profile for formulations showing $\mathrm{pH}$-dependent release or sustained release. Actually the main problem associated with the identification of most physiologically relevant medium is that only the relative and not the absolute concentration of SP in human epithelial LF is known [44].

Rohrschneider et al. performed an experiment to evaluate the Transwell ${ }^{\circledR}$ system for characterization of dissolution behavior of three corticosteroids (ciclesonide, budesonide and fluticasone propionate (FP)) differing in their lipophilicity. In this study, Andersen cascade impactor (ACI) and Next generator impactor (NGI) were used for sample collection. For dissolution study, PBS with $0.5 \%$ SDS was used as simulated RTLF dissolution medium for budesonide and ciclesonide, whereas PBS (pH 7.4) was used for FP. It was observed that in the absence of surfactant, less than $10 \% \mathrm{FP}$ dissolved over a period of $24 \mathrm{~h}$, indicating slow and incomplete dissolution. Analysis of dissolution profile for ciclesonide was unsuccessful because of its even lower solubility in medium without surfactant which resulted in concentrations below the limit of quantification of ultraviolet-high performance liquid chromatography. In case of budenoside, it was observed that about $90 \%$ of the drug dissolved in $24 \mathrm{~h}$ in presence of surfactant $(0.5 \% \mathrm{SDS})$ in the dissolution medium. It is to be kept in mind that the composition of LF is altered in pathological conditions, when it becomes challenging for the conventionally used media to mimic the composition of physiological LF. Hence, the development of a "fit for purpose" simulant demonstrating physiological relevance becomes essential [45]. Some of the commonly used simulated RTLF for in vitro dissolution study of inhaled drugs (for example, salbutamol (SB), voriconazole, fluticasone, itraconazole (ITZ), budenoside, and clarithromycin) include distilled deionized water, combination of PBS + Polyethylene oxide (PEO) + L-alpha-phosphatidylcholine, combination of Survanta ${ }^{\mathrm{TM}}+$ Normal Saline, PBS + Tween 80, Citrate buffer + ascorbic acid and PBS + PEO [46-49].

May et.al. performed an experiment to investigate factors affecting dissolution of powders for pulmonary delivery with USP apparatus 2 (paddle apparatus) and ACI was employed for dose collection. Budenoside was used as model drug and mixture of PBS (pH 7.4) and $0.02 \%$ DPPC was employed as RTLF medium. Addition of $0.02 \%$ DPPC improved the wettability of active pharmaceutical ingredient, resulting in better discrimination of dissolution profiles and good reproducibility as confirmed by the fit factor test [48]. Haghi et al. performed another experiment to investigate and compare deposition, dissolution and transport of salbutamol sulfate (SS) and free SB inhalation powders by employing Franz diffusion cell. It was observed that release of salbutamol base was relatively slow with time for $50 \%$ drug diffusion $\left(t_{50}\right)$ at 120 min whereas diffusion of SS was fast with a $t_{50}$ of $30 \mathrm{~min}$. Such observations are predictable and suggest that DPPC has little impact on dissolution behavior of SS since this drug is freely soluble in aqueous HBSS and SLF, compared to the slightly soluble SB. Subsequently, it is evident that SS undergoes faster wetting, and therefore, a faster dissolution and diffusion in the Franz cell apparatus. Addition of DPPC to SLF has been shown to improve wetting of hydrophobic drug particles [50].

A variety of fluids that have been used through past 30 years as dissolution media to mimic the LFs for the assessment of inhaled drug products include water, salt solutions having similar composition to that of PBS (pH 7.4), Gamble's solution of low pH, Eagle's basal medium, Hanks' Buffered Salt Solution (HBSS), etc. Gamble's solution, which contains $\mathrm{Mg}^{2+}, \mathrm{Ca}^{2+}, \mathrm{Na}^{+}, \mathrm{Cl}^{-}, \mathrm{HCO}_{3}{ }^{-}$, citrate ions, amino acids, antioxidants, Polysorbate (Tween) 80 or natural surfactant in different concentrations was developed to mimic the interstitial fluid of the lung. PBS contains ingredients such as $\mathrm{NaCl}(8.77 \mathrm{~g} / \mathrm{l}), \mathrm{Na}_{2} \mathrm{HPO}_{4}(1.28 \mathrm{~g} / \mathrm{l})$, and $\mathrm{KH}_{2} \mathrm{PO}_{4}(1.36 \mathrm{~g} / \mathrm{l})$ resulting in $\mathrm{pH}$ of $7.3 \pm 0.1$. Hanks' Buffered Salt Solution contains ingredients such as $\mathrm{NaCl}, \mathrm{KCl}, \mathrm{CaCl}_{2}, \mathrm{MgSO}_{4} \cdot 7 \mathrm{H}_{2} \mathrm{O}$, $\mathrm{MgCl}_{2} \cdot 6 \mathrm{H}_{2} \mathrm{O}, \mathrm{Na}_{2} \mathrm{HPO}_{4} \cdot 2 \mathrm{H}_{2} \mathrm{O}, \mathrm{KH}_{2} \mathrm{PO}_{4}$, D-glucose (Dextrose), and $\mathrm{NaHCO}_{3}[51-53]$. The actual LF contains all the ions mentioned in case of HBBS but in addition LF also contains ions such as citrate, acetate and proteins such as glyco-mucoproteins and various SP. Survanta ${ }^{\circledR}$ is another conventionally used medium which contains phospholipids $(25 \mathrm{mg} / \mathrm{ml})$, triglycerides $(0.5-1.75 \mathrm{mg} / \mathrm{ml})$, free fatty acids $(1.4-$ $3.5 \mathrm{mg} / \mathrm{ml}$ ), and protein content less than $1.0 \mathrm{mg} / \mathrm{ml}$. Eedara et.al. performed an experiment on in vitro dissolution testing of respirable size anti-tubercular drug (moxifloxacin and ethionamide) particles using flow perfusion cell as dissolution apparatus and modified Twin Stage Impinger as sample collection apparatus. Dissolution behavior of the fine particle dose was studied at either various perfusate flow rates $\left(0.2,0.4\right.$, and $0.8 \mathrm{~mL} \mathrm{~min}^{-1}$ of PBS) keeping mucus simulant concentration fixed at $1.5 \% \mathrm{w} / \mathrm{v}$ PEO (MW of $5000 \mathrm{kDa}$ ) in PBS (pH 7.4), or in media containing varying concentrations of mucus simulant (1.0, 1.5 and $2 \% \mathrm{w} / \mathrm{v}$ ) maintaining constant flow rate of $0.4 \mathrm{~mL} \mathrm{~min}^{-1}$ and in the presence of LS such as Curosurf [46]. Curosurf is a surfactant containing PC and two hydrophobic SP-B and SP-C. It was concluded that in presence of the Curosurf surfactant the dissolution profile of the two mentioned drugs mimicked more closely to the in vivo dissolution profile. The limitation of using conventional medium is the fact that it does not contain SP due to which such medium fails to predict the in vivo dissolution profile of inhaled drugs accurately.

\section{BIO-RELEVANT DISSOLUTION MEDIUM IN STUDY OF PULMONARY DOSAGE FORMS}

Bio-relevant is a term, used to describe a medium that has relevance to the in vivo dissolution condition for the compound. The factors that need to be considered while developing the bio-relevant dissolution medium for pulmonary dosage forms include the hydrodynamic conditions of respiratory fluid, physicochemical characteristics of drug, predictability of plasma profile and lastly establishing in vitro-in vivo correlation (IVIVC). Artificial simulated RTLFs are often used for in vitro dissolution studies to determine the solubility and dissolution of inhaled drug particles. However, an important criterion to develop such appropriate media includes the ease of manufacturing the media in a simple, standardized and cost-effective manner. Truly predictive dissolution medium should correctly simulate in vivo dissolution in the lung. Data obtained with such media in dissolution studies should be accurate, reproducible and amenable to validation [54].

To establish the IVIVC relationship for inhaled drugs, it is crucial to develop a bio-relevant simulated LF (SLF) with well-defined composition. The SLF contains DPPC, di palmitoyl phosphatidyl glycerol (DPPG), cholesterol, 
albumin, immunoglobulin (IgG), transferrin, and antioxidants. Freshly made SLF has pH 7.2, viscosity of $1.138 \times 10^{-3}$ Pa.s, conductivity of $14.5 \mathrm{~ms}$, surface tension of $54.9 \mathrm{mN} / \mathrm{m}$, and density of $0.999 \mathrm{~g} / \mathrm{cm}^{3}[28]$. The main ingredients of SLF include 1,2-dipalmitoyl-sn-glycero-3-phosphocholine and 1,2-dipalmitoyl-sn-glycero-3-phospho-(1'-rac-glycerol) sodium salt (DPPG), purified human IgG, lyophilized human serum albumin, transferrin, cholesterol, ascorbate, urate, glutathione and gentamicin solution and HBSS. There are mainly five types of SLF used as biorelevant media for in vitro dissolution study of pulmonary dosage forms and are designated as SLF 1, SLF2, SLF3, SLF4 and SLF5 [55]. Detailed composition of five different types of SLF has been enlisted in Table 1. Among all these types of SLF, mainly SLF 3 and SLF 4 are used in drug release study. SLF2 represents a modified version of Gamble's solution which was used to estimate potential human exposure to mercury due to inhalation of airborne calcine particulates from mine waste [56]. SLF3 models the interstitial fluid where ionic equivalent amount of citrate was used to replace protein to avoid foaming. Care must be taken in mixing the components to prevent precipitation while preparing SLF 3. SLF 3 was used to evaluate the in vitro release of insulin from poly(lactideco-glycolide) (PLGA)/cyclodextrin porous particles intended for its pulmonary delivery. Insulin release was found to be biphasic with an initial burst followed by a controlled release of $85 \%$ of entrapped insulin in 10 days [57]. SLF4 was used to characterize in vitro release of ITZ from nebulized nanoparticle dispersions. In this study, ACI was used for sample collection and ITZ nanoparticles were found to dissolve rapidly in simulated LF. Nebulized aqueous colloidal dispersions of itraconazole nanoparticles exhibited aerodynamic characteristics suitable for deep lung delivery. An in vivo single-dose $24 \mathrm{~h}$ pharmacokinetics study of the nebulized ITZ nanoparticle dispersion demonstrated substantial lung deposition and systemic absorption with blood levels reaching a peak of $1.6 \mu \mathrm{g} / \mathrm{mL}$ serum in $2 \mathrm{~h}$. The particles dissolved rapidly in simulated LF to produce super-saturation levels up to 27 times of equilibrium solubility (Ceq) for the crystalline form [58].

\section{DISSOLUTION STUDY APPARATUSES FOR PDFS}

Dose or sample collection technique is a critical step that needs to be carefully considered to obtain an accurate, precise and reproducible dissolution profile of inhaled products. For sample collection, the European Pharmacopeia describes four apparatuses (identified as A, C, D and E) while the United States Pharmacopeia USP describes six apparatuses identified with numbers from 1 to $6[59,60]$. The apparatuses which are used for sample/dose collection before carry out the in vitro dissolution testing of pulmonary dosage forms include ACI (Apparatus D as per EU Pharmacopeia or Apparatus 1 as per USP), ACI with pre-
separator(Apparatus 3 as per USP), Glass twin impinger (Apparatus A as per EU Pharmacopeia), Marple-Miller impactor (Apparatus 2 as per USP), Multi-stage liquid impinger (Apparatus $\mathrm{C}$ as per EU Pharmacopeia or Apparatus 4 as per USP), NGI(Apparatus E as per EU Pharmacopeia or Apparatus 5 as per USP), and NGI without pre-separator (Apparatus 6 as per USP) [60]. Following sample collection, conventional apparatuses used to study dissolution profile of pulmonary dosage forms include the compendial paddle apparatus, flow-through apparatus, diffusioncontrolled cell systems, namely Transwell ${ }^{\circledR}$ system, and Dissolvit ${ }^{\circledR}$ system. In USP Type 2 apparatus, drug release from particles suitable for inhalation is assessed by directly dispersing the powder in dissolution medium. However, drawbacks associated with the use of paddle apparatus include adherence of the particles to the apparatus surfaces, difficulties in achieving a homogenous dispersion, poor wettability, and floating of the particle. Due to these drawbacks, modified paddle over disk apparatus has been employed. In the case of paddle over disk apparatus, a stainless support disk is placed under the paddle and the membrane filter used to collect the respirable fraction can be directly positioned on top of the support. The main advantage of this technique is that different types of aerosol particle collection filters in different filter holders can be used as an integral part of paddle apparatus. Examples of drug substances that have been studied using paddle apparatus include budesonide, fenoterol, hydrocortisone, disodium cromoglycate, etc. [61,62]. The flow-through cell apparatus known as USP Type 4 apparatus consists of cylindrical cell with a filter placed at the top. The dissolution medium is pumped through the cell from a reservoir. Small glass beads are placed at the bottom of the cell and membrane used to collect the particles from inhaler is placed on the beads. The advantages of this apparatus include its suitability for poorly soluble drugs and ability to maintain sink condition. Examples of drugs that have been investigated using flow-through cell apparatus include FP, budesonide, and triamcinolone acetonide [63]. In Transwell apparatus, a membrane filter is placed on the top of the membrane and NGI is employed for collection of deposited drug particles. The principal advantage of such system is the fact that very low volume of dissolution medium on the donor chamber is used in comparison to receiver chamber which enables the opportunity to mimic the limited volume of LF $[64,65]$. Examples of drugs which have been tested using Transwell apparatus include beclomethasone, ciclesonide, budesonide, and triamcinolone acetonide. In case of DissolvIt system, a glass surface called as Precise Inhale is used for the deposition of aerosol particles which is then brought into contact with simulated LF mucus made up of PEO gel. This system is thermostatically maintained at $37^{\circ} \mathrm{C}$. Real-time dissolution of particles can be monitored by optical microscope. The advantage of Dissolvit system is that it can simulate the physiological conditions

Table 1: Composition on five different types of SLF [55]

\begin{tabular}{|c|c|c|c|c|c|}
\hline Ingredients & SLF 1 (g/l) & SLF2 (mg/l) & SLF3 (g/l) & SLF4 (g/l) & SLF5 (mMol/l) \\
\hline Magnesium chloride & 0.095 & & 0.2033 & 0.2033 & \\
\hline Sodium chloride & 6.019 & 6800 & 6.0193 & 6.0193 & 116 \\
\hline Potassium chloride & 0.298 & & 0.2982 & 0.2982 & \\
\hline Disodium hydrogen phosphate & 0.126 & 1700 & & & \\
\hline Sodium sulfate & 0.063 & & 0.0710 & 0.0710 & \\
\hline Calcium chloride dihydrate & 0.368 & 290 & 0.3676 & 0.3676 & 0.2 \\
\hline Sodium acetate & 0.574 & 580 & 0.9526 & 0.9526 & \\
\hline Sodium hydrogen carbonate & 2.604 & 2300 & 2.6043 & 2.6043 & 27 \\
\hline Citric acid & & 420 & & & \\
\hline Glycine & & 450 & & & 5 \\
\hline Ammonium chloride & & 5300 & & & 10 \\
\hline Phosphoric acid & & 1200 & & & \\
\hline Sodium carbonate & & 630 & & & \\
\hline Potassium hydrogen phthalate & & 200 & & & \\
\hline Sulfuric acid & & 510 & & & 0.5 \\
\hline Sodium citrate dihydrate & & 590 & & & \\
\hline Sodium phosphate monobasic monohydrate & & & 0.1420 & 0.1420 & 1.2 \\
\hline L-Cysteine hydrochloride & & & & & 1.0 \\
\hline DPPC* & & & & $0.02 \%(w / v)$ & \\
\hline DTPA* & & & & & 0.2 \\
\hline ABDCB* & & & & & 50 \\
\hline
\end{tabular}

DPPC: Dipalmitoyl phosphatidyl choline; DTPA: Diethylenetriaminepentaacetic acid; ABDCB: Alkylbenzyldimethyl ammonium chloride 
in lungs more closely in comparison to methods thereby enabling the monitoring of both permeation and dissolution process. The limitation to this system is that it can simulate the absorption kinetics in trachea and larger bronchi rather than transport across thinner barriers in rest of bronchi and alveoli [47]. Budesonide, fluticasone are the examples of drugs which were characterized by employing Dissolvit system.

Suitability of different types of apparatuses used for drug release study varies with the type of pulmonary dosage forms. While flow-through cells were deemed more suitable to conduct the dissolution of nanoparticles compared to the basket or the paddle methods, Franz diffusion cell was preferred for characterization of drug release from polymer microparticles, compared to the faster dissolution rates obtained using the paddle and flow-through methods. When only the respirable fraction of the drugs was evaluated, the paddle apparatus with membrane holder produced more reproducible data compared to flow-through apparatus and Franz cells. Most of the methods described belong to agitated (stirred) systems [66-68]. Caution regarding agitation is required because an increase in flow rate causes an increase in dissolution rate in flow-through cells or as the stirring speed of either paddles or baskets is increased in their respective apparatus. To facilitate low-dose dissolution, some of the apparatuses have been adapted with either mini baskets or paddles $[69,70]$. The Transwell system, in particular, involves very small volumes for dissolution testing in which maintaining sink conditions could be challenging especially for poorly soluble particles.

It is essential to consider the type of formulation that needs to be tested while selecting the appropriate apparatus to carry out the dissolution study of inhaled drug products [71-73]. Based on the type of formulation, these apparatuses should be used for the quantification of the total mass of the drug released, the amount of drug collected in each location of the apparatus, and the aerodynamic PSD (APSD) of the inhaled product.

\section{CONCLUSION}

Dissolution testing is employed for in vitro assessment and prediction of the in vivo behavior of a pulmonary dosage form. Therefore, selection of appropriate dissolution medium which can mimic the physiological LF more closely, especially during pathological condition is an important step in design and development of dissolution study protocol for pulmonary dosage forms. Conventional media devoid of lung SP and specific salts failed to produce desirable results capable of predicting in vivo performance. Bio-relevant dissolution medium and appropriate dissolution apparatus may achieve drug release data in vitro that can predict in vivo behavior of pulmonary dosage forms. In this context, simulated LF of definite composition has been found to achieve the goal of developing in vitro dissolution study protocol with satisfactory level of relevance to physiological LF and can thus be regarded as biorelevant dissolution medium for characterization of pulmonary dosage forms, suitable for establishing IVIVC in later stages of dosage form development.

\section{AUTHORS' CONTRIBUTION}

The authors have contributed equally to content designing, writing and editing of manuscript.

\section{AUTHORS FUNDING}

No funding received from any source.

\section{CONFLICT OF INTEREST}

No conflict of interest by authors.

\section{REFERENCES}

1. Azarmi S, Roa WH, Lobenberg R. Targeted delivery of nanoparticles for the treatment of lung diseases. Adv Drug Deliv Rev 2008;60:863-75

2. Sung JC, Pulliam BL, Edwards DA. Nanoparticles for drug delivery to the lungs. Trends Biotechnol 2007;25:563-70.

3. Jaafar-Maalej C, Elaissari A, Fessi H. Lipid-based carriers: Manufacturing and applications for pulmonary route. Expert Opin Drug Deliv 2012;9:1111-27.

4. Beck-Broichsitter M, Gauss J, Packhaeuser CB, Lahnstein K, Schmehl T, Seeger W, et al. Pulmonary drug delivery with aerosolizable nanoparticles in an ex vivo lung model. Int J Pharm 2009;367:169-78.

5. Beck-Broichsitter M, Kleimann P, Gessler T, Seeger W, Kissel T, Schmehl T. Nebulization performance of biodegradable sildenafilloaded nanoparticles using the Aeroneb ${ }^{\circledR}$ Pro: Formulation aspects and nanoparticle stability to nebulization. Int J Pharm 2012;422:398-408.

6. Paranjpe M, Muller-Goymann S. Nanoparticle-mediated pulmonary drug delivery: A review. Int J Mol Sci 2014;15:5853-6.

7. David E, Geller MD. Comparing clinical features of the nebulizer, metered-dose inhaler and dry powder inhaler. Respir Care 2005;50:1313-21.

8. Anderson PJ. History of aerosol therapy: Liquid nebulization to MDIs to DPIs. Respir Care 2005;50:1139-49.

9. Rau JL. The inhalation of drugs: Advantages and problems. Respir Care 2005;50:367-82.

10. Everard ML. Inhaler devices in infants and children: Challenges and solutions. J Aerosol Med 2004;17:186-95.

11. Thorsson L, Geller D. Factors guiding the choice of delivery device for inhaled corticosteroids in the long-term management of stable asthma and COPD: Focus on budesonide. Respir Med 2005;99:836-49.

12. Dolovich MB, Ahrens RC, Hess DR, Anderson P, Dhand R, Rau JL, et al. Device selection and outcomes of aerosol therapy: Evidence based guidelines. Chest 2005;127:335-71.

13. Rau JL. Design principles of liquid nebulization devices currently in use. Respir Care 2002;47:1257-75.

14. Nikander K, Turpeinen M, Wollmer P. The conventional ultrasonic nebulizer proved inefficient in nebulizing a suspension. J Aerosol Med 1999; 12:47-53.

15. Hendeles L, Hatton RC, Coons TJ, Carlson L. Automatic replacement of albuterol nebulizer therapy by metered-dose inhaler and valved holding chamber. Am J Health Syst Pharm 2005;62:1053-61.

16. Kumar A, Terakosolphan W, Hassoun M, Vandera KK, Novicky A, Harvey R, et al. A biocompatible synthetic lung fluid based on human respiratory tract lining fluid composition. Pharm Res 2017;34:2454-65.

17. Pham S, Wiedmann TS. Dissolution of aerosol particles of budesonide in Survanta ${ }^{\mathrm{TM}}$, a model lung surfactant. J Pharm Sci 2001;90:98-104.

18. Son YJ, McConville JT. Preparation of sustained release rifampicin microparticles for inhalation. J Pharm Pharmacol 2012;64:1291-302.

19. Widmaier E, Raff H, Strang KT. Vander's Human Physiology. $10^{\text {th }}$ ed. New York: McGraw-Hill; 2005.

20. Grippi MA. Pulmonary Pathophysiology. $1^{\text {st }}$ ed. Philadelphia, PA: Lippincott, Williams and Wilkins; 1995.

21. Epstein SK. Anatomy and physiology of tracheostomy. Respir Care 2005;50:476-82.

22. Ahmed-Nusrath A, Tong JL, Smith JE. Pathways through the nose for nasal intubation: A comparison of three endotracheal tubes. $\mathrm{Br} \mathrm{J}$ Anaesth 2008;100:269-74.

23. Minnich DJ, Mathisen DJ. Anatomy of the trachea, carina, and bronchi. Thorac Surg Clin 2007;17:571-85.

24. Webb EM, Elicker BM, Webb WR. Using CT to diagnose nonneoplastic tracheal abnormalities: Appearance of the tracheal wall. AJR Am J Roentgenol 2000;174:1315-21.

25. Ugalde P, Miro S, Frechette E, Deslauriers J. Correlative anatomy for thoracic inlet; glottis and subglottis; trachea, carina, and main bronchi; lobes, fissures, and segments; hilum and pulmonary vascular system; bronchial arteries and lymphatics. Thorac Surg Clin 2007;17:639-59.

26. Haskin PH, Goodman LR. Normal tracheal bifurcation angle: A reassessment. AJR Am J Roentgenol 1982;139:879-82.

27. Widdicombe J. Regulation of the depth and composition of airway surface liquid. J Anat 2002;201:313-8.

28. Radivojev S, Zellnitz S, Paudel A, Fröhlich E. Searching for physiologically relevant in vitro dissolution techniques for orally inhaled drugs. Int J Pharm 2019;556:45-56.

29. Guagliardo R, Perez-Gil J, De Smedt S, Raemdonck K. Pulmonary surfactant and drug delivery: Focusing on the role of surfactant proteins. J Contr Release 2018;291:116-26.

30. Bastacky J, Lee CY, Goerke J, Koushafar H, Yager D, Kenaga L, et al. Alveolar lining layer is thin and continuous: Low-temperature scanning electron microscopy of rat lung. J Appl Physiol 1995;79:1615-28.

31. Lindert J, Perlman CE, Parthasarathi K, Bhattacharya J. Chloridedependent secretion of alveolar wall liquid determined by opticalsectioning microscopy. Am J Respir Cell Mol Biol 2007;36:688-96. 
32. Bhattacharya S, Glucksberg MR, Bhattacharya J. Measurement of lung microvascular pressure in the intact anesthetized rabbit by the micropuncture technique. Circ Res 1989;64:167-72.

33. Lai-Fook SJ. Perivascular interstitial fluid pressure measured by micropipettes in isolated dog lung. J Appl Physiol 1982;52:9-15.

34. Ying X, Qiao R, Ishikawa S, Bhattacharya J. Removal of albumin microinjected in rat lung perimicrovascular space. J Appl Physiol 1994; $77: 1294-302$

35. Nicolaysen G, Staub NC. Time course of albumin equilibration in interstitium and lymph of normal mouse lungs. Microvasc Res 1975;9:29-37

36. Hollenhorst M, Richter K, Fronius M. Ion transport by pulmonary epithelia. J Biomed Biotechnol 2011;15:1-9.

37. Manzanares D, Gonzalez C, Ivonnet P, Chen RS, Valencia-Gattas M, Conner GE, et al. Functional apical large conductance, $\mathrm{Ca}^{2+-}$ activated, and voltage dependent $\mathrm{K}^{+}$channels are required for maintenance of airway surface liquid volume. J Biol Chem 2011;286:19830-9.

38. Mall M, Bleich M, Greger R, Schreiber R, Kunzelmann K. The amiloride-inhibitable $\mathrm{Na}^{+}$conductance is reduced by the cystic fibrosis transmembrane conductance regulator in normal but not in cystic fibrosis airways. J Clin Invest 1998;102:15-21

39. Burch LH, Talbot CR, Knowles MR, Canessa CM, Rossier BC, Boucher RC. Relative expression of the human epithelial $\mathrm{Na}^{+}$channel subunits in normal and cystic fibrosis airways. Am J Physiol 1995;269:511-8.

40. Schwiebert EM, Kizer N, Gruenert DC, Stanton BA. GTP-binding proteins inhibit cAMP activation of chloride channels in cystic fibrosis airway epithelial cells. Proc Natl Acad Sci U S A 1992;89:10623-7.

41. Mitchell JP, Nagel MW. Cascade impactors for the size characterization of aerosols from medical inhalers: Their use and limitations. J Aerosol Med 2003;16:341-77.

42. Uddin MD, Hossain MD, Mamun AA, Zaman S, Asaduzzaman M, Rashid M, et al. Pharmacopoeial standards and specifications for pharmaceutical aerosols: In-process and finished products quality control tests. Adv Res 2016;6:1-12.

43. Anand O, Yu LX, Conner DP, Davit BM. Dissolution testing for generic drugs: An FDA perspective. AAPS J 2011;13:328-35.

44. Hamm H, Kroegel C, Hohlfield J. Surfactant: A review of its function and its relevance in adult respiratory disorders. Respir Med 1996;90:251-70.

45. Rohrschneider M, Bhagwat S, Krampe R, Michler V, Breitkruetz J, Hocchaus G. Evaluation of the transwell system for characterization of dissolution behavior of inhalation drugs: Effects of membrane and surfactant. Mol Pharm 2015;12:2618-24.

46. Eedara BB, Tucker IG, Das SC. In vitro dissolution testing of respirable size anti-tubercular drug particles using a small volume dissolution apparatus. Int J Pharm 2019;559:235-44.

47. Gerde P, Malmlof M, Havsborn L, Sjöberg CO, Ewing P, Eirefelt S, et al. DissolvIt: An in vitro method for simulating the dissolution and absorption of inhaled dry powder drugs in the lungs. Assay Drug Dev Technol 2017; 15:77-88.

48. May S, Jensen B, Weiler C, Wolkenhauer M, Schneider M, Lehr CM. Dissolution testing of powders for inhalation: Influence of particle deposition and modeling of dissolution profiles. Pharm Res 2014;31:3211-24.

49. Arora S, Haghi M, Loo CY, Traini D, Young PM, Jain S. Development of an inhaled controlled release voriconazole dry powder formulation for the treatment of respiratory fungal infection. Mol Pharm 2015;12:2001-9.

50. Haghi M, Traini D, Bebawy M, Young PM. Deposition, diffusion and transport mechanism of dry powder microparticulate salbutamol at the respiratory epithelia. Mol Pharm 2012;9:1717-26.

51. Arora D, Shah KA, Halquist MS, Sakagami M. In vitro aqueous fluidcapacity-limited dissolution testing of respirable aerosol drug particles generated from inhaler products. Pharm Res 2010;27:786-95.

52. Pritchard RJ, Ghio AJ, Lehmann JR, Winsett DW, Tepper JS, Park P, et al. Oxidant generation and lung injury after particulate air pollutant exposure increase with the concentrations of associated metals. Inhal Toxicol 1996;8:457-77.
53. Vallyathan V, Pack D, Leonard S, Lawson R, Schenker M, Castranova V. Comparative in vitro toxicity of grape and citrus farm dusts. J Toxicol Environ Health 2007;70:95-106.

54. Bhagat NB, Yadav AV, Mali SS, Khutale RA, Hajare AA. A review on development of biorelevant dissolution medium. J Drug Deliv Ther 2014;4:140-8.

55. Margareth RC, Loebenberg R, Almukainzi M. Simulated biological fluids with possible application in dissolution testing. Dissolut Technol 2011;18:15-28.

56. Gray JE, Plumlee GS, Morman SA, Higueras PL, Crock JG, Lowers HA, et al. In vitro studies evaluating leaching of mercury from mine waste calcine using simulated human body fluid. Environ Sci Technol 2010;44:4782-8

57. Ungaro F, Bianca RE, Giovino C, Miro A, Sorrentino R, Quaglia F, et al. Insulin-loaded PLGA/cyclodextrin large porous particles with improved aerosolization properties: In vivo deposition and hypoglycemic activity after delivery to rat lungs. J Control Release 2009;135:25-34

58. Yang W, Tam J, Miller DA, Zhou J, McConville JT, Johnston KP, et al. High bioavailability from nebulized itraconazole nanoparticle dispersions with biocompatible stabilizers. Int J Pharm 2008;361:177-88.

59. European Pharmacopoeia. Preparations for inhalation: Aerodynamic assessment of fine particles. In: European Pharmacopoeia, 8e, 309-320. Strasbourg, France: European Directorate for the Quality of Medicines, Council of Europe; 2014

60. US Pharmacopeia. Aerosols, Nasal Sprays, Metered-dose Inhalers and Dry Powder Inhalers, USP 37 NF 32. Rockville, MD: US Pharmacopeia; 2014.

61. Salama RO, Traini D, Chan HK, Young PM. Preparation and characterisation of controlled release co-spray dried drug-polymer microparticles for inhalation: Evaluation of in vitro release profiling methodologies for controlled release respiratory aerosols. Eur J Pharm Biopharm 2008;70:145-52.

62. Duret C, Wauthoz N, Sebti T, Vanderbist F, Amighi K. Solid dispersions of itraconazole for inhalation with enhanced dissolution, solubility and dispersion properties. Int J Pharm 2012;428:103-13.

63. Davies NM, Feddah MR. A novel method for assessing dissolution of aerosol inhaler products. Int J Pharm 2003;255:175-87.

64. May S, Kind S, Jensen B, Wolkenhauer M, Schneider M, Lehr CM. Miniature in vitro dissolution testing of powders for inhalation. Dissolut Technol 2015;8:40-51.

65. Lewis DA, Young PM, Buttini F, Church T, Colombo P, Forbes B, et al. Towards the bioequivalence of pressurized metered dose inhalers: Design and characterization of aerodynamically equivalent beclomethasone dipropionate inhalers with and without glycerol as a non-volatile excipient. Eur J Pharm Biopharm 2013;3486:31-7.

66. Vengala P, Vanamala R. Nanocrystal technology as a tool for improving dissolution of poorly soluble drug, lornoxicam. Int J Appl Pharm 2018; $10: 162-8$

67. Rahul S, Abhinand CR, Nikithareddy B, Jayachandra K, Lakshmi P, Doddayya $\mathrm{H}$, et al. Pharmacoeconomic evaluation of acute exacerbations of chronic obstructive pulmonary disease at a tertiary care teaching hospital in North Karnataka, India. Asian J Pharm Clin Res 2018;11:463-6.

68. Panda N, Reddy V, Reddy GV, Sultana A. Formulation design and in vitro evaluation of bilayer sustained release matrix tablets of doxofylline. Int J Pharm Pharm Sci 2015;7:74-83.

69. Anwar N, Jan S, Gul R. Formulation and evaluation of glibenclamide gel for transdermal drug delivery. Int J Curr Pharm Res 2020;12:35-9.

70. Alshargabi A. In vitro assessment of physicochemical parameters of five generics amlodipine besylate tablets marketed in Yemen. Int $\mathrm{J}$ Pharm Pharm Sci 2021;13:15-9.

71. May S, Jensen B, Wolkenhauer M, Schneider M, Lehr CM. Dissolution techniques for in vitro testing of dry powders for inhalation. Pharm Res 2012;29:2157-8

72. Bhattacharyya SN, Wesley JA, Fioritto A, Martin PJ, Babu SR Dissolution testing of a poorly soluble compound using the flowthrough cell dissolution apparatus. Int J Pharm 2002;236:135-7.

73. Heng D, Cutler DJ, Chan HK, Yun J, Raper JA. What is a suitable dissolution method for drug nanoparticles? Pharm Res 2008;25:1696-8. 